\title{
Time-Dependent Density Functional Theory for Driven Lattice Gas Systems with Interactions
}

\author{
Marcel Dierl ${ }^{1,2}$, Philipp Maass ${ }^{2}$, and Mario Einax ${ }^{1,2}$ \\ ${ }^{1}$ Institut für Physik, Technische Universität Ilmenau, 98684 Ilmenau, Germany \\ 2 Fachbereich Physik, Universität Osnabrück, \\ Barbarastraße 7, 49076 Osnabrück, Germany
}

(Dated: March 1, 2022)

\begin{abstract}
We present a new method to describe the kinetics of driven lattice gases with particle-particle interactions beyond hard-core exclusions. The method is based on the time-dependent density functional theory for lattice systems and allows one to set up closed evolution equations for mean site occupation numbers in a systematic manner. Application of the method to a totally asymmetric site exclusion process with nearest-neighbor interactions yields predictions for the current-density relation in the bulk, the phase diagram of non-equilibrium steady states and the time evolution of density profiles that are in good agreement with results from kinetic Monte Carlo simulations.
\end{abstract}

PACS numbers: 05.50.+q, 05.60.Cd, 05.70.Ln 
Driven lattice gases are an active topic in non-equilibrium statistical mechanics due to both their manifold applications and their importance in fundamental studies of nonequilibrium systems (for reviews, see [1 5 5]). Prominent examples for applications to nonequilibrium processes in nature are biopolymerization [6], unidirectional motions of motor proteins along filaments or microtubuli [7, 8], flow of molecules through vessels or ion conduction through membrane channels [9, 10], incoherent transport of electrons in molecular wires [11], surface growth [12] and traffic [13]. With respect to fundamental aspects, questions pertaining to the theoretical description of boundary-induced phase transitions and of nonequilibrium steady states (NESS) can be studied within a conceptually simple framework $[2,3]$.

A standard model of a driven lattice gas system is the asymmetric simple exclusion process (ASEP), which refers to the directional stochastic hopping transport of particles on a one-dimensional lattice with hard-core exclusions that prevent multiple occupation of a lattice site. In an open system with particle injections and ejections at the left and right boundaries, this model exhibits phase transitions between non-equilibrium steady states of different mean site occupation in the bulk [14, 15]. Analytical methods like the matrix approach or Bethe ansatz have been developed and successfully applied to calculate exactly the distribution of microstates in the NESS [2 5]. Various extensions of the ASEP have been considered in the past, as, for example, particle rods covering several lattice sites [16], or stochastic injection/ejection (Langmuir kinetics) of particles in the bulk [17]. So far, however, comparatively few studies exist [14, 18, 19] which are concerned with the description of driven lattice gas systems, where the particles do not only interact via athermal hard-core exclusion.

In this work we will focus on developing a method for treating the kinetics of such systems with interactions beyond hard-core exclusions. In general, starting with the master equation for the Markovian time evolution of the microstates, exact evolution equations can be derived for the mean site occupation numbers (henceforth called densities). These have the form of a continuity equation with currents depending on equal-time correlations of occupation numbers (henceforth called "correlators"). The challenge is to develop proper methods to calculate these correlators so that closed evolution equations for the densities result that well account for the kinetic behavior. In the treatment of interaction effects in the driven system considered by Schütz et al. [19] this problem was addressed by utilizing 
the special jump rates introduced in the Katz-Lebowitz-Spohn model [18], which yield a site occupation statistics in a driven bulk system that can be exactly mapped onto an equilibrium system with nearest-neighbor interactions (Ising system). As a consequence, the currentdensity relation in the bulk could be determined exactly and, by employing the maximum and minimum current principle [14, 19], the phase diagram of NESS in an open system calculated in good agreement with Monte Carlo simulations.

In this Letter we show how by using the time-dependent density functional theory (TDFT) for lattice systems a systematic method is provided that allows one to treat the kinetics of driven lattice gases with interactions. To demonstrate the procedure, we consider a totally asymmetric site exclusion process (TASEP) with nearest-neighbor interactions. Predictions of the theory for the current-density relation in the bulk, the phase diagram of NESS, the density profiles in the NESS, and the time evolution of density profiles are compared to results of kinetic Monte Carlo (KMC) simulation and yield a surprisingly good agreement for this far-from-equilibrium system.

The Hamiltonian for a one-dimensional lattice gas with nearest-neighbor interactions $V$ is

$$
\mathcal{H}(\boldsymbol{n})=V \sum_{i} n_{i} n_{i+1}
$$

The set of occupation numbers $\boldsymbol{n}=\left\{n_{i}\right\}$ specifies the microstate of the system, where $n_{i}=0$ or 1 if site $i$ is vacant or occupied by a particle, respectively $\left(n_{i}^{2}=n_{i}\right)$. For unidirectional nearest-neighbor hopping considered in the TASEP, the stochastic dynamics of the system is specified by the rates $\Gamma_{i}(\boldsymbol{n})$ for a particle on site $i$ to jump to a vacant neighboring site $(i+1)$ in the configuration $\boldsymbol{n}$. Starting from the master equation for the time evolution of the probability density $P(\boldsymbol{n}, t)$ of microstates, one derives the discrete version of the continuity equation for the densities $\rho_{i}(t)=\left\langle n_{i}\right\rangle_{t}$ (see, e. g. [20] for a general derivation),

$$
\frac{d \rho_{i}(t)}{d t}=j_{i-1}(t)-j_{i}(t)
$$

where the mean currents from $i$ to $i+1$ are given by

$$
j_{i}(t)=\left\langle n_{i}\left(1-n_{i+1}\right) \Gamma_{i}(\boldsymbol{n})\right\rangle_{t},
$$

and $\langle\cdots\rangle_{t}$ denotes an average with respect to $P(\boldsymbol{n}, t)$. To complete the specification of the dynamics we define the jump rates $\Gamma_{i}(\boldsymbol{n})$ as [22]

$$
\Gamma_{i}(\boldsymbol{n})=\nu \exp \left[-\left(\mathcal{H}\left(\boldsymbol{n}^{(i, i+1)}\right)-\mathcal{H}(\boldsymbol{n})\right) / 2 k_{\mathrm{B}} T\right] .
$$


Here $\nu$ is an attempt frequency, and $\boldsymbol{n}^{(i, i+1)}$ refers to the target configuration of the jump, where, with respect to the initial configuration $\boldsymbol{n}, n_{i}$ and $n_{i+1}$ are interchanged, while the other $n_{k}$ are the same. We use $\nu^{-1}$ as time unit and $k_{\mathrm{B}} T$ as energy unit in the following $\left(\nu^{-1}=1\right.$ and $\left.k_{\mathrm{B}} T=1\right)$. With Eq. (4) the currents can be written explicitely as

$j_{i}=\left\langle\tilde{n}_{i-1} n_{i} \tilde{n}_{i+1} \tilde{n}_{i+2}\right\rangle_{t}+e^{V / 2}\left\langle n_{i-1} n_{i} \tilde{n}_{i+1} \tilde{n}_{i+2}\right\rangle_{t}+e^{-V / 2}\left\langle\tilde{n}_{i-1} n_{i} \tilde{n}_{i+1} n_{i+2}\right\rangle_{t}+\left\langle n_{i-1} n_{i} \tilde{n}_{i+1} n_{i+2}\right\rangle_{t}$,

where we introduced the hole occupation numbers $\tilde{n}_{i}=1-n_{i}$.

In order to express the correlators in Eq. (51) in terms of the densities, we now apply the TDFT, which is based on the (time-)local equilibrium approximation. According to this, $P(\boldsymbol{n}, t)$ is expressed by the Boltzmann probability associated with $\mathcal{H}(\boldsymbol{n})$ plus a timedependent external potential $U[\boldsymbol{n}, \boldsymbol{\rho}(t)]=\sum_{i} v_{i}[\boldsymbol{\rho}(t)] n_{i}$ that equals the potential which would generate $\boldsymbol{\rho}(t)=\left\{\rho_{i}(t)\right\}$ as equilibrium density profile (according to the classical version of the Mermin theorem this potential is unique for given interaction). In effect this implies that the correlators at any time $t$ are supposed to be related to the densities as in an equilibrium system.

To find the equilibrium correlator-density relations, we apply the methods developed in the Markovian approach to derive exact density functionals [21]. Accordingly we express the joint probabilities $p_{\mathrm{eq}}^{(j+1)}\left(n_{i}, \ldots, n_{i+j}\right)$ for the occupation numbers $n_{i}, \ldots, n_{i+j}$ in equilibrium by the Markov chain $p_{\mathrm{eq}}^{(j+1)}\left(n_{i}, \ldots, n_{i+j}\right)=p_{\mathrm{eq}}^{(1)}\left(n_{i}\right) \prod_{s=1}^{j} w\left(n_{i+s} \mid n_{i+s-1}\right)$, where $p_{\mathrm{eq}}^{(1)}\left(n_{i}\right)$ is the equilibrium probability for $n_{i}$, and $w\left(n_{i+1} \mid n_{i}\right)=p_{\mathrm{eq}}^{(2)}\left(n_{i}, n_{i+1}\right) / p_{\mathrm{eq}}^{(1)}\left(n_{i}\right)$ the conditional probability for $n_{i+1}$ if $n_{i}$ is given. Since the joint probabilities are directly connected to the correlators, e. g., $p_{\text {eq }}^{(4)}\left(n_{i-1}=0, n_{i}=1, n_{i+1}=0, n_{i+2}=1\right)=\left\langle\tilde{n}_{i-1} n_{i} \tilde{n}_{i+1} n_{i+2}\right\rangle_{\text {eq }}$, all correlators involving more than two occupation numbers in Eq. (5) can thus be reduced to two-point correlators. The TDFT expression for the current hence becomes

$$
j_{i}^{\mathrm{TDFT}}=\left[\left(\rho_{i+2}-C_{i+1}\right) e^{-V / 2}+\tilde{\rho}_{i+1}-\rho_{i+2}+C_{i+1}\right] \frac{\rho_{i}-C_{i}}{\rho_{i} \tilde{\rho}_{i+1}}\left[\rho_{i}-C_{i-1}+e^{V / 2} C_{i-1}\right],
$$

with $\tilde{\rho}_{i}=1-\rho_{i}$ and $C_{i}=\left\langle n_{i} n_{i+1}\right\rangle_{t}$. The two-point correlators are related to the densities via [21]

$$
C_{i}=\exp (-V) \frac{\left(\rho_{i}-C_{i}\right)\left(\rho_{i+1}-C_{i}\right)}{1-\rho_{i}-\rho_{i+1}+C_{i}},
$$

which can be explicitly solved to yield functions $C_{i}=C_{i}\left(\rho_{i}, \rho_{i+1}\right)$. In this way, the currents $j_{i}^{\mathrm{TDFT}}$ are given as functionals of the density profile $\boldsymbol{\rho}(t)$. 
To test the quality of the TDFT approach we start by considering a homogeneous system with periodic boundary conditions. In this case we suppress the site indices in Eq. (7) and set $j_{i}=j(\rho)$ in Eq. (6) . For $V \rightarrow 0, j(\rho)$ approaches the parabola $j=\rho-\rho^{2}$ for particles feeling only hard-core repulsion. When $V$ exceeds a critical value $V_{\mathrm{c}}=-2 \ln (\sqrt{5}-2) \simeq 2.89, j(\rho)$ develops a double-hump structure with two maxima at densities $\rho_{1,2}^{*}=\rho_{1,2}^{*}(V)$. In the limit $V \rightarrow \infty$ we find $\rho_{1,2}^{*}=1 / 2 \pm(\sqrt{2}-1) / 2$, in agreement with earlier findings reported by $\operatorname{Krug}$ [14], and $j \rightarrow\left(x^{3 / 2}-2 x+x^{1 / 2}\right) /(1-x)$ with $x=(2 \rho-1)^{2}$, meaning that the particle movement is frozen for a half-filling system.

In Fig. 1 we compare the analytical findings (a) for the pair correlator $C(\rho)$ and (b) the current-density relation $j(\rho)$ with results from KMC simulations for various $V$. For $V \lesssim V_{\text {c }}$ as well as for large $V \gtrsim 4 V_{\mathrm{c}}$, we find an excellent agreement with the simulation data, see also the inset in Fig. 1(a), which shows the interaction dependence of $C(\rho=0.5)$. For intermediate interaction strength $V_{\mathrm{c}}<V<4 V_{\mathrm{c}}$ and near half-filling, some deviations occur. An interesting effect is seen in Fig. 1(b) for weak couplings $V \lesssim V_{\mathrm{c}}$ : both the theoretical predictions and the simulations show an increased current compared to the case $V=0$. This phenomenon is caused by effective particle-hole attraction for weak coupling strengths.

Next we consider an open system of $N$ sites that is coupled to two particle reservoirs L and $\mathrm{R}$ at the left and right boundaries with densities $\rho_{\mathrm{L}}$ and $\rho_{\mathrm{R}}$, respectively. The dynamics of injection (ejection) of particles from the left (right) reservoir is defined such that the same correlator-density relations apply at the boundaries as in the corresponding bulk (periodic ring) systems with densities $\rho_{\mathrm{L}}$ and $\rho_{\mathrm{R}}$. We used the method described in [19] to implement this model in the KMC simulations.

Let us first see how well the TDFT approach captures the time evolution of density profiles. To this end we consider reservoir densities $\rho_{\mathrm{L}}=0.4$ and $\rho_{\mathrm{R}}=0.1$ and an initially empty lattice system at time $t=0$. For the interaction we choose deliberately $V=2 V_{\mathrm{c}}$, where comparatively large deviations were seen in Fig 1. Such a "worst-case" choice permits the best evaluation of approximation limits. In Fig. 2 we compare the results from numerical solutions of the nonlinear rate equations (2) [where the $j_{i}$ are given by Eqs. (66) and (17)] with the $\mathrm{KMC}$ results for four different times and in the stationary limit $t \rightarrow \infty$. The excellent agreement between the two data sets demonstrates the power of our TDFT approach for driven lattice gas systems with interactions. In the inset of Fig. 2 we show the steady state $(t \rightarrow \infty)$ profile for the corresponding non-interacting case $V=0$. As one would expect 
from an application of the maximum and minimum current principle to the bulk currentdensity relation shown in Fig. 1(b), different bulk densities $\rho_{\mathrm{B}}$ are found for the two different interaction strengths: $\rho_{\mathrm{B}}=\rho_{\mathrm{L}}=0.4$ for $V=0$ and $\rho_{\mathrm{B}}=\rho_{1}^{*}\left(2 V_{\mathrm{c}}\right) \simeq 0.31$ for $V=2 V_{\mathrm{c}}$.

More generally, using the minimum and maximum current principle, we can evaluate the phase diagram of the NESS for arbitrary interaction strengths based on the bulk currentdensity relation derived above. Alternatively we can use the $t \rightarrow \infty$ limit of the rate equations to identify the bulk densities of the NESS. As expected, we find that the results of both procedures agree [23]. As an example we show in Fig. 3(a) the phase diagram of the NESS for $V=2 V_{\mathrm{c}}$, and for each of the occurring seven phases we display the stationary density profile in Fig. 3(b). In all cases the predictions are well confirmed by the KMC results. The largest deviations occur for the transition lines between the phases I/III, I/VII and the "mirror lines" IV/II and IV/VII. Note that the phase diagram in Fig. 33(a) exhibits particle-hole symmetry as required by the Hamiltonian in Eq. (1) and the dynamics specified in Eq. (4). There are two maximal current phases V and VI characterized by the bulk densities $\rho_{\mathrm{B}}=\rho_{1}^{*}$ and $\rho_{\mathrm{B}}=\rho_{2}^{*}$, respectively, and one minimal current phase with $\rho_{\mathrm{B}}=0.5$. The remaining four phases are determined by the reservoir densities $\rho_{\mathrm{L}}$ and $\rho_{\mathrm{R}}$.

In summary, we presented a new analytical approach to describe the kinetics of driven lattice gas systems with interactions beyond hard-core exclusions based on the TDFT. The approach was demonstrated for a TASEP with nearest-neighbor interaction and provided very good results for the kinetic behavior. It is clear that the TDFT is not restricted to the TASEP situation or nearest-neighbor interactions, but can be applied also to ASEPs (or purely boundary driven systems) as well as other types of interactions. In the TDFT closed nonlinear evolution equations for the densities result due to local relations between correlators and densities, which allow one to capture the kinetics much better than simple factorization schemes (note that simple factorization schemes of correlators would completely fail for $V>V_{\mathrm{c}}$ in the example studied in this work). Although one can regard it as a weakness, it is, on the hand, advantageous that the TDFT relies on density functionals for equilibrium systems. The development and improvement of such equilibrium functionals in condensed matter systems have been an intensive research area in the past, in particular also with respect to find good approximation schemes for the setup of functionals in dimensions larger than one. Hence one can expect that these developments will be useful to treat more complex non-equilibrium systems. We hope that our findings will stimulate further research 
in this direction.

We thank W. Dieterich for very valuable discussions.

[1] B. Schmittmann and R. K. P. Zia, in Phase Transitions and Critical Phenomena, edited by C. Domb and J. L. Lebowitz (Academic Press, London, 1995), Vol. 17.

[2] B. Derrida, Phys. Rep. 301, 65 (1998).

[3] G. M. Schütz, in Phase Transitions and Critical Phenomena, edited by C. Domb and J. L. Lebowitz (Academic Press, San Diego, 2001), Vol. 19.

[4] O. Golinelli and K. Mallick, J. Phys. A 39, 12679 (2006).

[5] R. A. Blythe and M. R. Evans, J. Phys. A 40, R333 (2007).

[6] C. T. MacDonald, J. H. Gibbs, and A. C. Pipkin, Biopolymers 6, 1 (1968).

[7] R. Lipowsky, S. Klumpp, and T. M. Nieuwenhuizen, Phys. Rev. Lett. 87, 108101 (2001).

[8] K. Nishinari, Y. Okada, A. Schadschneider, and D. Chowdhury, Phys. Rev. Lett. 95, 118101 (2005).

[9] B. Hille, Ionic Channels of Excitable Membranes, 3rd ed. (Sinauer Associates, Sunderland, MA, 2001).

[10] M. Einax, M. Körner, P. Maass, and A. Nitzan, Phys. Chem. Chem. Phys. 12, 645 (2010).

[11] C. Joachim and M. A. Ratner, Proc. Natl. Acad. Sci. USA 102, 8801 (2005).

[12] J. Krug and H. Spohn, in Solids far from Equilibrium, edited by C. Godrèche (Cambridge University Press, Cambridge, 1991).

[13] D. Helbing, Rev. Mod. Phys. 73, 1067 (2001).

[14] J. Krug, Phys. Rev. Lett. 67, 1882 (1991).

[15] B. Derrida, E. Domany, and D. Mukamel, J. Stat. Phys. 69, 667 (1992).

[16] G. Lakatos and T. Chou, J. Phys. A 36, 2027 (2003).

[17] A. Parmeggiani, T. Franosch, and E. Frey, Phys. Rev. Lett. 90, 086601 (2003).

[18] S. Katz, J. L. Lebowitz, and H. Spohn, J. Stat. Phys. 34, 497 (1984).

[19] J. S. Hager, J. Krug, V. Popkov, and G. M. Schütz, Phys. Rev. E 63, 056110 (2001).

[20] J.-F. Gouyet, M. Plapp, W. Dieterich, and P. Maass, Adv. Phys. 52, 523 (2003).

[21] J. Buschle, P. Maass, and W. Dieterich, J. Phys. A 33, L41 (2000).

[22] This choice is motivated by the fact that these rates agree with the forward rates of an 
ASEP, which fulfills detailed balance conditions with respect to the equilibrium Boltzmann distribution in a non-driven system.

[23] We note that the TDFT allows one to treat general boundary couplings, where the correlatordensity relations at the system boundaries can be different from those in the bulk. In this case the maximum/minimum current principle cannot be applied generally since the stationary density profiles become non-monotonous. This, however, is not the focus of the present work and will be discussed in detail elsewhere. 

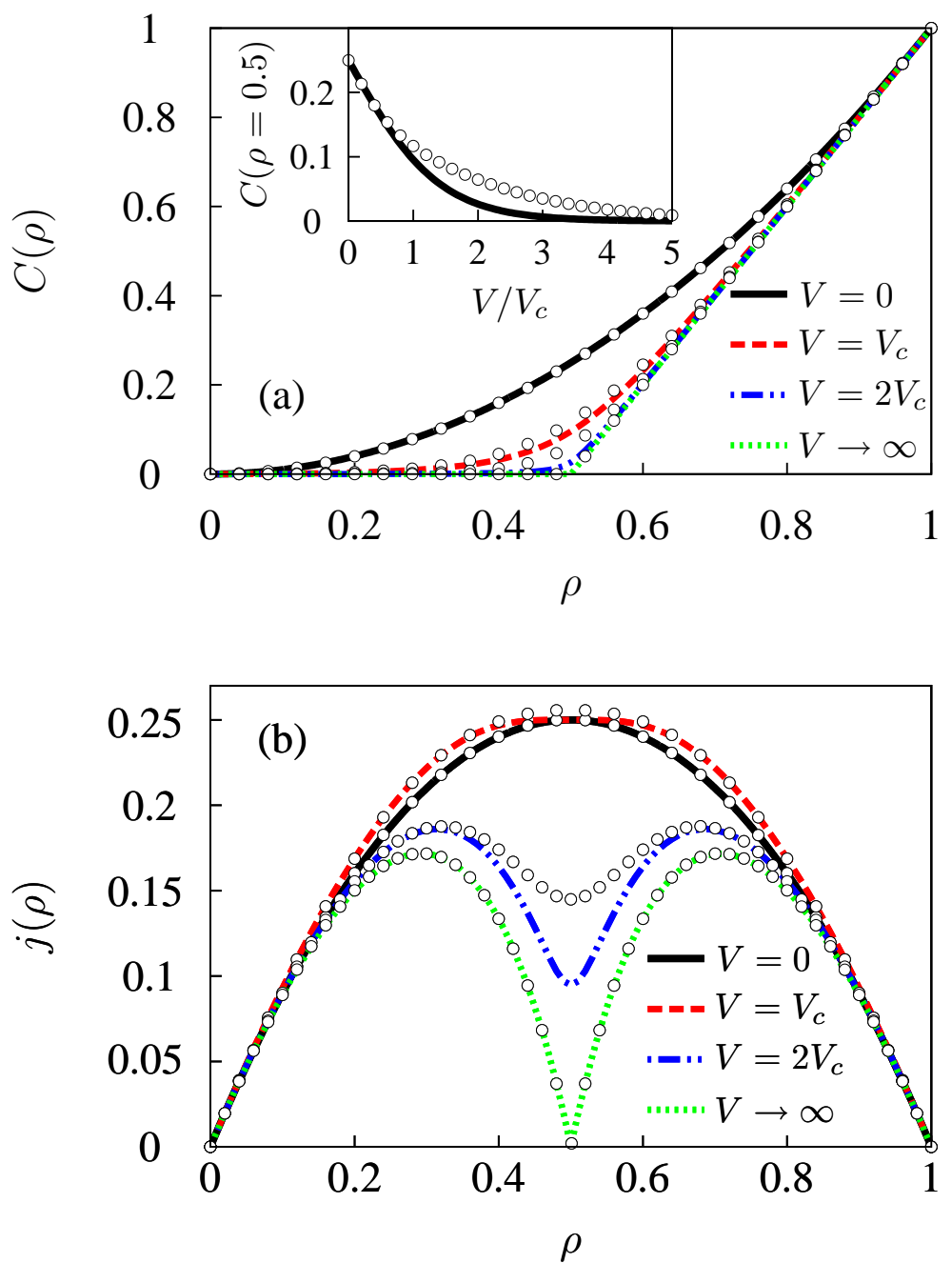

FIG. 1. (Color online) (a) Two-point correlator $C(\rho)$ and (b) current-density relation $j(\rho)$ for various $V$ calculated by Eqs. (6) and (17) (lines). The inset in Fig. 1(a) shows the pair correlator as a function of $V / V_{\mathrm{c}}$ for fixed density $\rho=0.5$. The analytical results are compared with the true non-equilibrium quantities obtained by Monte Carlo simulations (circles) for a ring system with 1000 sites. Each data point results from an average over a time corresponding to $10^{9}$ particle jumps in the steady state. 


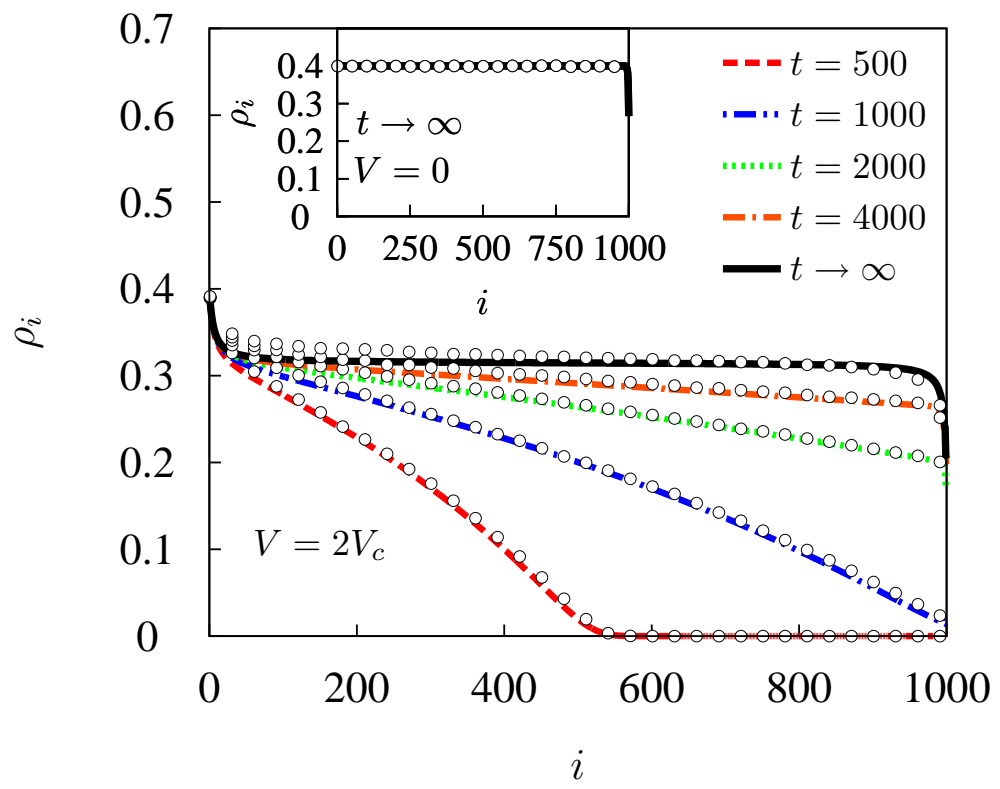

FIG. 2. (Color online) Time evolution of $\rho_{i}$ from an initially empty lattice with $\rho_{\mathrm{L}}=0.4, \rho_{\mathrm{R}}=0.1$, $V=2 V_{\text {c }}$ and $N=1000$. TDFT solutions are marked by lines. The KMC results (circles) are averaged over $10^{6}$ configurations. For $V=0$, the steady-state density profile is shown in the inset. 
(a)

(b)
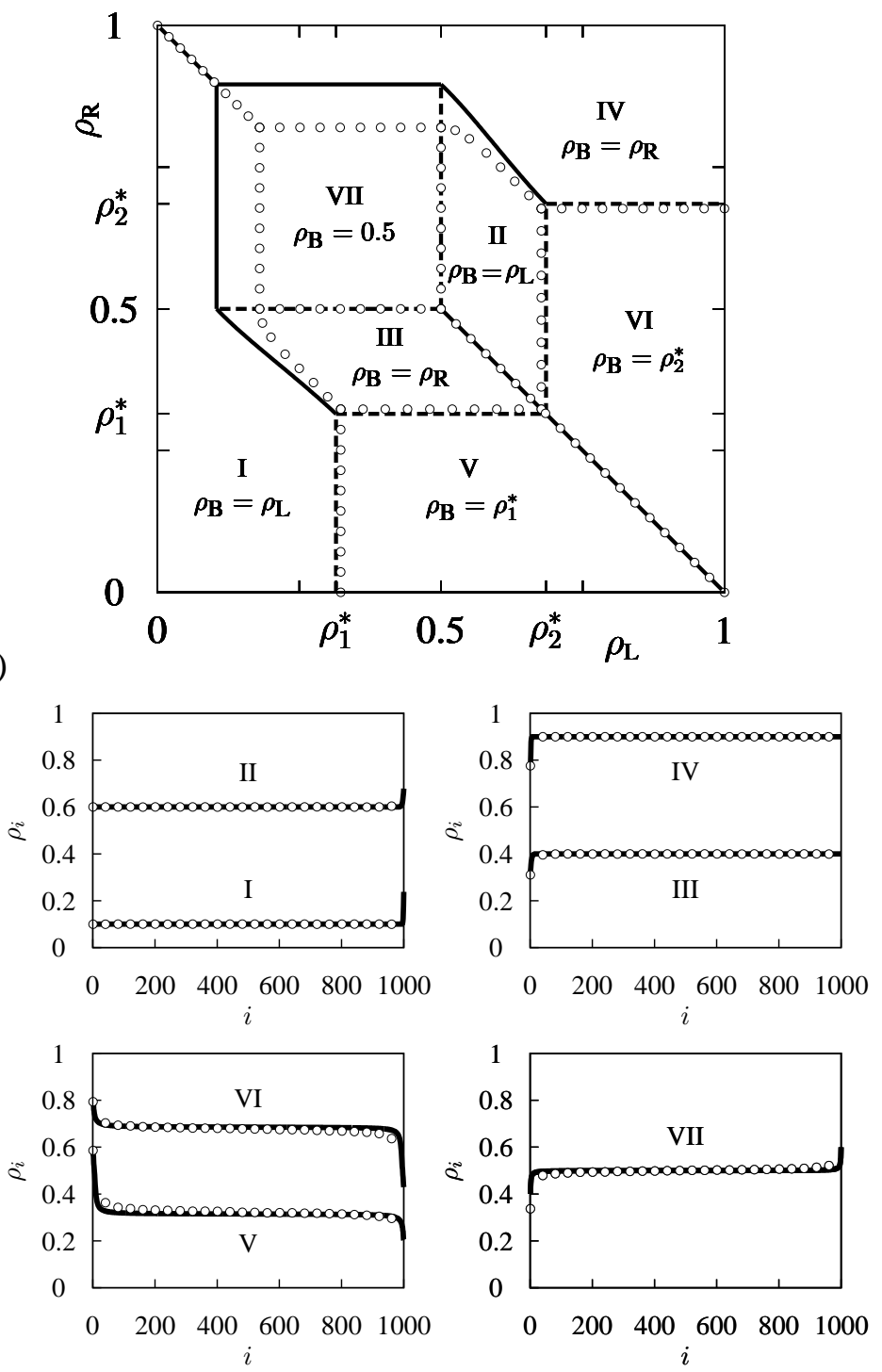

FIG. 3. (a) Steady-state phase diagram and (b) density profiles of a system with 1000 sites and $V=2 V_{\mathrm{c}}$. The phase diagram contains seven phases which are represented by density profiles corresponding to I $\left(\rho_{\mathrm{B}}=\rho_{\mathrm{L}}\right.$, low-density phase): $\rho_{\mathrm{L}}=0.1, \rho_{\mathrm{R}}=0.4$; II $\left(\rho_{\mathrm{B}}=\rho_{\mathrm{L}}\right): \rho_{\mathrm{L}}=0.6$, $\rho_{\mathrm{R}}=0.7 ;$ III $\left(\rho_{\mathrm{B}}=\rho_{\mathrm{R}}\right): \rho_{\mathrm{L}}=0.3, \rho_{\mathrm{R}}=0.4 ; \mathrm{IV}\left(\rho_{\mathrm{B}}=\rho_{\mathrm{R}}\right.$, high-density phase: $\rho_{\mathrm{L}}=0.6, \rho_{\mathrm{R}}=0.9$; $\mathrm{V}\left(\rho_{\mathrm{B}}=\rho_{1}^{*}\right.$, maximal-current phase $): \rho_{\mathrm{L}}=0.6, \rho_{\mathrm{R}}=0.1 ; \mathrm{VI}\left(\rho_{\mathrm{B}}=\rho_{2}^{*}\right.$, maximal-current phase): $\rho_{\mathrm{L}}=0.9, \rho_{\mathrm{R}}=0.4 ; \mathrm{VII}\left(\rho_{\mathrm{B}}=0.5\right.$, minimal-current phase $): \rho_{\mathrm{L}}=0.3, \rho_{\mathrm{R}}=0.7$. Solid and dashed lines in the phase diagram, which denote first- and second-order phase transitions, respectively, as well as density profiles are calculated using TDFT. Data points are obtained by Monte Carlo simulations with the same statistics as in Fig. 1. 